\title{
Polysplenia syndrome with a rare variation between the common hepatic artery and the superior mesenteric artery in adults
}

Jingjing Liu, Kai Yang, Jianbo Wang

Department of Interventional Radiology, The Sixth People's Hospital Affiliated to Shanghai Jiaotong University, Xuhui District, Shanghai, China

Submitted: 10 December 2018

Accepted: 23 March 2019

Arch Med Sci 2020; 16 (5): 1263-1266

DOI: https://doi.org/10.5114/aoms.2020.97972

Copyright $\odot 2020$ Termedia \& Banach

Polysplenia syndrome (PSS), a heterotaxy syndrome, is a rare congenital anomaly with a reported incidence of 1 in 250,000 live births and was first described by Helwig [1] in 1929. Most patients with PSS die in the early neonatal period because the disease is often accompanied by serve cardiac and biliary abnormalities [2]. However, some patients have only mild cardiovascular malformations or anomalies in the abdominal organs, which are typically diagnosed incidentally in adulthood [3]. Anomalies in the cardiovascular tract including bilateral superior vena cava, interruption of the inferior vena cava with azygos continuation, ventricular septal defect, ostium primum defect, and morphologic left ventricular outflow obstruction were present in at least $50 \%$ of 146 autopsied cases of polysplenia reviewed [4]. Preduodenal portal vein is another anomaly frequently associated with polysplenia syndrome and Rameshbabu et al. described this anomaly in 7 out of 19 cases of his own autopsied series [5]. PSS is a rare disease, and there is gradually increasing literature reporting the accompanying various abnormalities in order to make people realize what these accompanying abnormalities are. However, PSS with a variation between the common hepatic artery $(\mathrm{CHA})$ and the superior mesenteric artery (SMA) in adults is rarely reported in the literature. The CHA is a short blood vessel arising from the celiac trunk (CT) that supplies the liver, pylorus, pancreas, and duodenum [6]. The SMA arises from the anterior surface of the abdominal aorta, approximately $2 \mathrm{~cm}$ below the $C T$, and supplies the pancreas, duodenum, jejunum, ileum, cecum, appendix, and ascending colon, as well as part of the transverse colon [7].

We herein report on a patient who was diagnosed with PSS and was later found to have a vascular anatomical variation between the $\mathrm{CHA}$ and the SMA. It makes people realize that PSS may be accompanied by a new vascular variability and highlights the importance for anatomists, surgeons, and radiologists of the awareness of this easily missed and rare vascular anomaly in PSS due to its prominent significance in many clinical procedures, such as radiological abdominal intervention and abdominal surgery.

A 39-year-old Chinese woman with no prior medical history presented with intermittent abdominal pain for 6-7 days. She had no previous history of splenectomy, and physical examination revealed no abnormality. She underwent plain and contrast-enhanced upper abdominal computed tomography examinations, which revealed a malformation syndrome

\author{
Corresponding author: \\ Jianbo Wang \\ Department of \\ Interventional Radiology \\ The Sixth People's \\ Hospital Affiliated \\ to Shanghai Jiaotong \\ University \\ 600 Yishan Road \\ Xuhui District \\ Shanghai 200233, China \\ E-mail: jeanbob_wang@163. \\ com
}




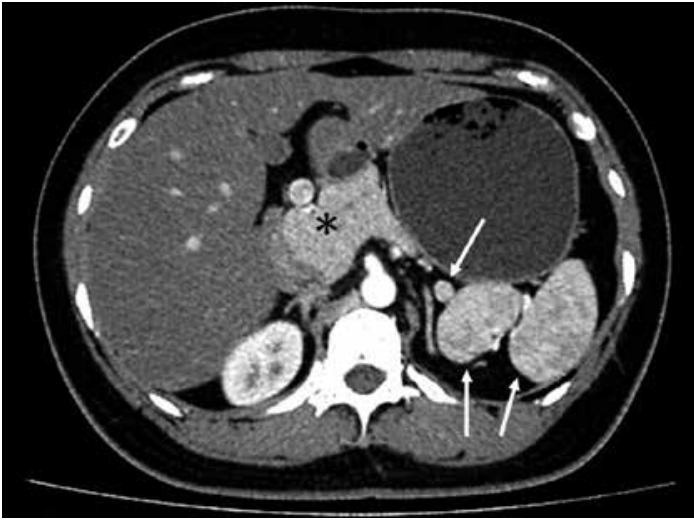

Figure 1. Axial contrast-enhanced computed tomographic image showing evidence of a malformation syndrome with multiple spleens (arrows) and a short pancreas (asterisk)

with multiple spleens and agenesis of the pancreatic tail (Figure 1). Images revealed multiple irregularly shaped nodules in the left upper abdomen and "spotted" enhancement in the arterial phase consistent with spleen enhancement. Computed tomography also revealed the absence of the hepatic segment of the inferior vena cava (IVC), with IVC continuation into the dilated azygos vein (Figure 2). Midgut dysplasia appeared in most of the small intestine in the right half of the abdomen and was absent in the left half of the abdomen (Figure 3).

Today, multi-slice spiral computed tomography angiography, which shows the blood vessels clearly after the contrast agent is filled, has great diagnostic and clinical value in vascular anomaly diseases. In addition, post-processing the image after angiography can produce a three-dimensional image. On detailed analysis of the images using three-dimensional computed tomography volume rendering technology, a rare anastomosis between the CHA and the SMA was observed (Figure 4). The $C T$ was divided into only two branches, the left gastric artery and the splenic artery, and the CHA arose anomalously from the SMA and was

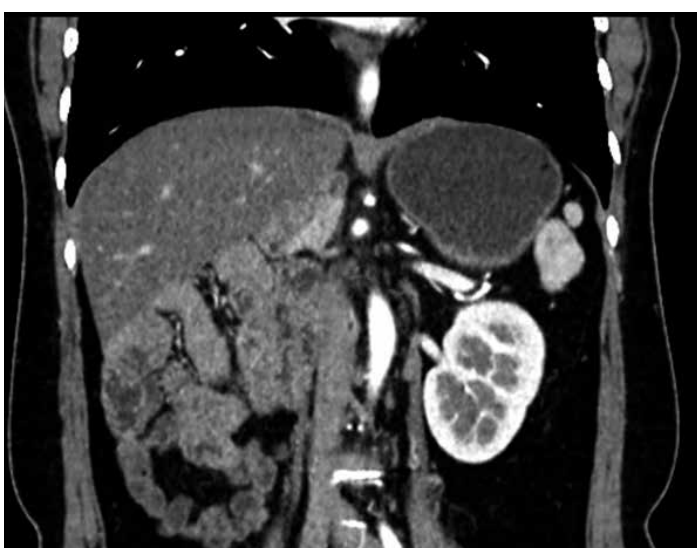

Figure 3. Coronal reformatted computed tomographic image demonstrating midgut dysplasia

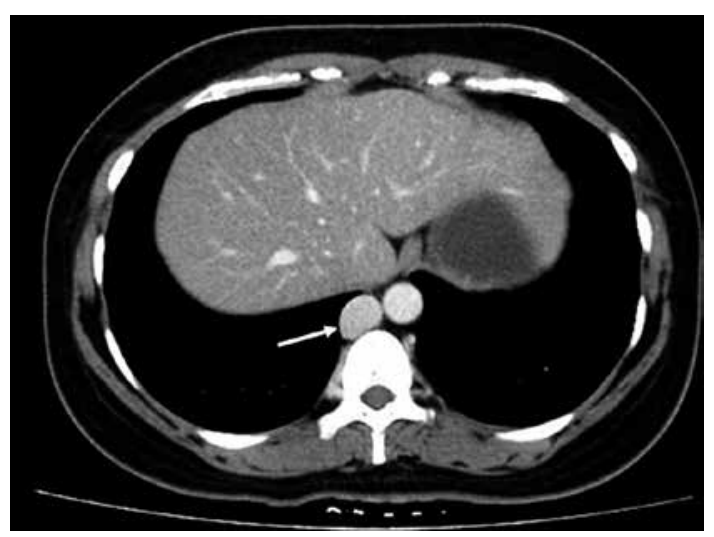

Figure 2. The hepatic segment of the inferior vena cava (IVC) was absent, with IVC continuation into the dilated azygos vein (arrow)

divided into the gastroduodenal artery (GDA) and the proper hepatic artery ( $\mathrm{PHA})$.

PSS is generally defined as the presence of multiple spleens, usually numbering between two and six, and various organ anomalies in the absence of a history of splenectomy [8]. PSS is also associated with multiple congenital variations that may involve the heart, solid organs, and digestive tube of the abdominal cavity, or the great vessels [9]. The spleen develops from the splenic primordia, originating from the dorsal mesogastrium, during the fifth embryonic week. The initial splenic primordia are then created as incisures on the left side of the dorsal mesogastrium; when the incisures fail to fuse, they create two or more spleens

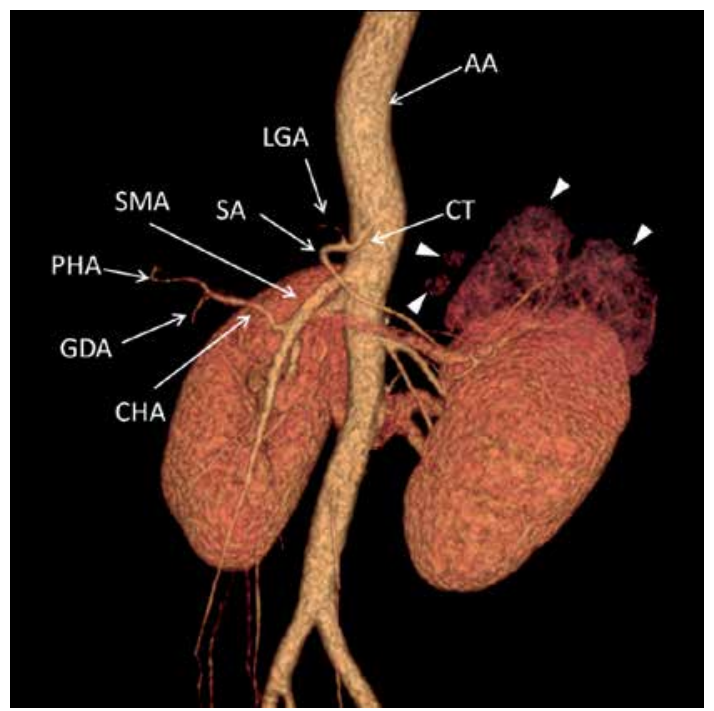

Figure 4. Three-dimensional volume-rendered computed tomographic image showing anastomosis between the common hepatic artery and the superior mesenteric artery (white arrowheads represent multiple spleens)

$A A$ - abdominal aorta, CT - celiac trunk, LGA - left gastric artery, SA - splenic artery, SMA - superior mesenteric artery, PHA - proper hepatic artery, GDA - gastroduodenal artery, CHA-common hepatic artery. 
[10]. As most affected adults do not exhibit symptoms, PSS is often diagnosed incidentally during other procedures, but fewer than $5 \%$ of patients with PSS remain alive at 5 years of age due to associated severe cardiac anomalies [2]. The most common vascular anomalies are agenesis of the suprarenal IVC with persistent continuity of the azygos vein and the preduodenal portal vein [11, 12]. Cases involving CHA and SMA variations, as in our patient, are recognized as very minor anomalous cases and are rarely described as vascular anatomical variations of PSS in the literature [13].

The CHA arises from the CT and is divided into the GDA and the PHA [6]. The SMA arises from the abdominal aorta, giving off five sets of branches: the inferior pancreaticoduodenal artery, the intestinal arteries, the ileocolic artery, the right colic artery, and the middle colic artery [7]. Previous case reports have also described other types of variation between the CHA and the SMA [14-16]. Kardile et al. [14] indicated that the CHA was sometimes absent, with the right accessory hepatic artery arising from the SMA. In a relatively rare variation, termed hepatomesenteric trunktype CHA, the CHA originates entirely as a branch of the SMA and no accessory left hepatic artery arises from the CT [15]. These variations can be explained embryonically: this change in the arterial system distributed to the digestive tube occurs before rotation of the gut begins. These variations arise from differences in the pattern of the partial disappearance or survival of the ventral splanchnic arteries and the ventral longitudinal channel.

PSS with a variation between the CHA and the SMA is often diagnosed incidentally in adults by computed tomography. Although this condition is rarely encountered, we cannot rule out the possibility of its presence in cases of clinically unexplained abdominal pain and gastrointestinal symptoms. The study has indicated that the variation of the CHA and the SMA may cause chronic mesenteric ischemia [17]; however, more cases are needed for statistical analysis to explore whether this variation could indeed cause intermittent abdominal pain. The variation also has important clinical significance in liver transplantation, abdominal laparoscopy, cephalic pancreaticoduodenectomy, and radiological abdominal intervention [17].

In conclusion, recognition of the unstable nature of all of these variations is extremely important. PSS has no uniform or characteristic clinical pattern, or typical laboratory findings. Most importantly, when upper abdominal or gastrointestinal surgery is required in a patient with PSS, care must be exercised and the surgeon must be highly familiar with normal anatomy to identify anatomical variations revealed in imaging studies to predict and limit intraoperative risks. Failure to detect the anatomical variation between the $\mathrm{CHA}$ and the SMA prior to surgery can result in severe complications, such as hemorrhage and vascular ligation. Such accidents can be prevented by careful preoperative diagnostic imaging examination, such as by computed tomography with contrast injection, and recognition of the possibility of an anatomical variation between the $\mathrm{CHA}$ and the SMA in cases of PSS.

\section{Conflict of interest}

The authors declare no conflict of interest.

\section{References}

1. Gayer G, Apetr S, Jonas T, et al. Polysplenia syndrome detected in adulthood: report of eight cases and review of the literature. Abdom Imaging 1999; 24: 178-84.

2. Gayer G, Hertz M, Strauss S, Zissin R. Congenital anomalies of the spleen. Semin Ultrasound CT MR 2006; 27 : 358-69.

3. Low JP, Williams D, Chaganti JR. Polysplenia syndrome with agenesis of the dorsal pancreas and preduodenal portal vein presenting with obstructive jaundice: a case report and literature review. Br J Radiol 2011; 84: e217-20.

4. Peoples WM, Moller JH, Edwards JE. Polysplenia: a review of 146 cases. Pediatr Cardiol 1983; 4: 129-37.

5. Rameshbabu CS, Gupta KK, Qasim M, Gupta OP. Heterotaxy polysplenia syndrome in an adult with unique vascular anomalies: case report with review of literature. J Radiol Case Rep 2015; 9: 22-37.

6. Araujo Neto SA, Franca HA, de mello Junior CF, et al. Anatomical variations of the celiac trunk and hepatic arterial system: an analysis using multidetector computed tomography angiography. Radiol Bras 2015; 48: 358-62.

7. Koplay M, Kantarci M. Common hepatic artery arising from the aorta - demonstration with multidetector CT angiography and its clinical importance. Arch Med Sci 2011; 7: 176-7.

8. De La Villeon B, Le Goudeveze A, Goudard Y, et al. Polysplenia syndrome. J Visc Surg 2011; 148: e395-6.

9. Varga I, Galfiova P, Adamkov M, et al. Congenital anomalies of the spleen from an embryological point of view. Med Sci Monit 2009; 15: Ra269-76.

10. Gayer G, Apter S, Jonas T, et al. CT findings in congenital anomalies of the spleen. Br J Radiol 2001; 74: 767-72.

11. Vanhoenacker FM, De Ruysscher D, De Backer Al, De Schepper AM. Heterotaxy syndrome in an adult, with polysplenia, visceral and cardiovascular malposition. JBR-BTR 2001; 84: 1-4.

12. Cheung CKM, Law MF, Wong KT, Tam MTK, Chow KM. Massive pulmonary embolism in a patient with polysplenia syndrome and interrupted inferior vena cava with azygous continuation. Arch Med Sci 2018; 14: 251-3.

13. Hasegawa T, Kimura T, Sasaki T, Okada A. Living-related liver transplantation for biliary atresia associated with polysplenia syndrome. Pediatr Transplant 2002; 6: 78-81.

14. Kardile PB, Ughade JM, Ughade MN, et al. Anomalous origin of the hepatic artery from the hepatomesenteric trunk. J Clin Diagn Res 2013; 7: 386-8.

15. Nakano H, Kikuchi K, Seta SI, et al. A patient undergoing pancreaticoduodenectomy in whom involved common hepatic artery anomalously arising from the superi- 
or mesenteric artery was removed and reconstructed. Hepatogastroenterology 2005; 52: 1883-5.

16. Chen J, Ramjit A, Ahmad N. Replaced gastroduodenal artery with continuation as accessory left hepatic artery: a rare anatomical variant. CVIR Endovasc 2018; 1: 23.

17. Chentanez V, Nateniyom N, Huanmanop T, Agthong S. Co-existence of the double inferior vena cava with complex interiliac venous communication and aberrant common hepatic artery arising from superior mesenteric artery: a case report. Folia Morphol 2018; 77: 151-5. 